\title{
Zur physikalischen Interpretation manifest kovarianter Darstellungen der inhomogenen Lorentzgruppe zur Masse Null
}

\author{
WERNER LANGBEIN \\ Physikalisches Institut der Universität Würzburg \\ Eingegangen am 25. November 1966
}

\begin{abstract}
It is shown, that the class of fields $(a, b)$ involving a particle of zero rest mass and helicity $h$ is restricted only by the condition $|a+b| \geqq|h|,\left(\frac{a+b}{h}\right.$ integer), if the representation space is not required to be irreducible. The main feature of the corresponding physical interpretation is the occurence of gauge particles. The investigations are carried out in terms of manifest covariant representations of the inhomogeneous Lorentz Group, which are shown to be not fully reducible in the zero rest mass case. The results are also obtained by a limiting process $m \rightarrow 0$.
\end{abstract}

\section{Einleitung}

Im Rahmen der Feldtheorie treten bekanntlich zwei Typen von Darstellungen der inhomogenen Lorentzgruppe (iLG) auf. Einmal die manifest kovarianten Darstellungen, die durch das Konzept lokaler Feldoperatoren und Wechselwirkungen in die Theorie kommen, zum anderen die unitären Darstellungen, die für eine relativistisch invariante Wahrscheinlichkeitsinterpretation unentbehrlich sind und eng mit den Teilchenobservablen zusammenhängen.

Bei vorgegebener Masse (Klein-Gordon-Gleichung) ist ihr Zusammenhang rein gruppentheoretischer Natur: Man hat es mit dem Problem zu zu tun, die manifest kovarianten Darstellungen auf eine unitäre Form zu bringen; die in der Theorie auftretenden Teilchen werden dann durch ihre irreduziblen Bestandteile beschrieben. Da die inhomogene Lorentzgruppe nicht kompakt ist, braucht eine solche Transformation nicht immer möglich zu sein. Dasselbe gilt natürlich auch für die vollständige Zerlegbarkeit in irreduzible Bestandteile, die bekanntlich erst durch die Unitarität garantiert wird (vgl. HAMMERMESH [1]).

Der Fall nichtverschwindender Ruhmasse $(m \neq 0)$ ist daraufhin wiederholt untersucht worden [2], [3]. Das Resultat ist einfach: Ein manifest kovariantes Feld des Typs ${ }^{1}(a, b)$ enthält Teilchen des Spins

$1(a, b)$ ist die durch die Spinorindizes des Feldes gegebene Darstellung der homogenen Lorentzgruppe.

6 Commun. math. Phys., Vol. 5 
$a+b, \ldots, a-b$, wobei die Unitarität durch Transformation auf Ruhe im Spinorraum und die Ausreduktion durch eine anschließende ClebschGordan-Umkopplung erreicht wird.

Im Falle $m=0$ liegen die Verhältnisse komplizierter. S. Weinberg [4] hat gezeigt, daß ein Teilchen der Helizität $h$ nur durch Felder des Typs $(a+h, a)$ beschrieben werden kann, wenn sein Zustandsraum unter der iLG irreduzibel ist. Auf der anderen Seite weiß man jedoch vom Photon, daß es aus Gründen der mathematischen Einfachheit ratsam sein kann, zur Formulierung der Wechselwirkungen des Teilchens Felder zu benutzen, die nicht zu dieser Klasse gehören. Es ist daher zumindest zur Konstruktion relativistisch invarianter Wechselwirkungen von Interesse, die Klasse der Felder zu erweitern, die einem Teilchen der Helizität $h$ zugeordnet werden können. Dazu streichen wir die Forderung der Irreduzibilität unter der iLG und untersuchen, welche Teilchen einer manifest kovarianten Darstellung der iLG, die lediglich durch die KleinGordon-Gleichung zur Masse Null eingeschränkt ist, zugeordnet werden können ${ }^{2}$.

Das mathematische Problem besteht offenbar darin, die Beziehungen zwischen den manifest kovarianten Darstellungen der iLG und ihren bekannten unitären Darstellungen zur Masse Null [5], [6] zu klären. Zu seiner Lösung bedienen wir uns der Methode der kleinen Gruppe [7]. Insbesondere läßt sich zeigen, daß zu einer manifest kovarianten Darstellung der iLG, deren Spinoranteil die infinitesimalen Erzeugenden $\boldsymbol{\Sigma}$ und $\boldsymbol{I}$ für Raumdrehungen bzw. Lorentztransformationen hat, eine Darstellung der kleinen Gruppe mit den infinitesimalen Erzeugenden:

$$
\boldsymbol{\Sigma}+\frac{\overline{\mathbf{p}}}{\bar{p}_{0}} \times \boldsymbol{\Pi}
$$

gehört. $\bar{p}=\left(\overline{\mathbf{p}}, \bar{p}_{0}\right)$ ist dabei der Normalviererimpuls, dessen Invarianz die kleine Gruppe definiert. Im Fall $m \neq 0$, d. h. $\overline{\mathbf{p}}=0 \bar{p}_{0}=m$ wird sie zur Drehgruppe und die verschiedenen Teilchen entsprechen ihren irreduziblen Darstellungen.

Ist jedoch $m=0$, so kann nur noch $\overline{\mathbf{p}}=\mathbf{e}_{z}, \bar{p}_{0}=1$ erreicht werden, was die Struktur der Darstellungen wesentlich ändert. Sie bleiben zwar im allgemeinen reduzibel, jedoch nicht mehr vollreduzibel. Es zeigt sich vielmehr, daß sie bestenfalls auf eine Form gebracht werden können, in der auf der einen Seite der Hauptdiagonalen stets Nullen stehen, während die Hauptdiagonale selbst aus Phasenfaktoren besteht. Die zugehörige Basis wird dabei von den gemeinsamen Eigenzuständen $|p h n\rangle$ von $P_{\mu}, \boldsymbol{\Sigma} \cdot \mathbf{P}$ und $\boldsymbol{\Pi} \cdot \mathbf{P}$ gebildet; bei Lorentztransformationen $L$ geht ein Zustand $|p h n\rangle$ in eine Superposition von Zuständen $\left|L p h^{\prime} n^{\prime}\right\rangle$ mit

${ }^{2}$ Da vollreduzible Darstellungen auf irreduzible zurückgeführt werden können, bedeutet dies im wesentlichen die Hinzunahme „halbreduzibler" Darstellungen. 
$n^{\prime} \geqq n$ über, wobei der zu $n^{\prime}=n$ gehörige Anteil aus $|L p h n\rangle$ durch Multiplikation mit einem Phasenfaktor hervorgeht. Dadurch wird einerseits eine relativistisch invariante Wahrscheinlichkeitsinterpretation möglich, zum anderen aber auch die Rolle der Nebenbedingungen und Eichfelder für $m=0$ Teilchen klar. Definiert man nämlich das Skalarprodukt lediglich durch die zu einem festen Index $n=N$ gehörigen Amplituden, so ist die Lorentzinvarianz gesichert, sobald die zu $n<N$ gehörigen Amplituden durch einen Satz entsprechender Nebenbedingungen ausgeschlossen sind. Die zu $n>N$ gehörigen Amplituden transformieren sich zwar bei Lorentztransformationen nicht unitär, sie liefern jedoch per definitionem zu diesem Skalarprodukt keine Beiträge und können deshalb als Eichzusätze aufgefaßt werden. Derartige Verhältnisse sind ja vom elektromagnetischen Feld her bekannt. Es zeigt sich, daß ein Feld des Typs $(a, b)$ auf diese Weise Teilchen der Helizitäten $|a+b|, \ldots,-|a+b|$ beschreiben kann, wenn man nur jeweils die Nebenbedingungen geeignet wählt.

Die Überlegungen sind in drei Teile gegliedert. In einem ersten untersuchen wir die Transformation der manifest kovarianten Darstellungen auf die korrespondierenden Darstellungen der kleinen Gruppe für die Fälle $m \neq 0$ und $m=0$, und in einem zweiten die spezifischen Eigenschaften dieser Darstellungen bei $m=0$ sowie die damit verknüpfte Teilcheninterpretation. In einem dritten Teil wird gezeigt, daß die Ergebnisse auch durch einen Grenzübergang $m \rightarrow 0$ erreicht werden können.

\section{Manifest kovariante Darstellungen der inhomogenen Lorentzgruppe und ihre Zurückführung auf Darstellungen der kleinen Gruppe}

Eine relativistisch invariante Quantentheorie zeichnet sich bekanntlich dadurch aus, daß sich die inhomogene Lorentzgruppe im Raum der physikalischen Zustände durch eine Gruppe linearer Operatoren $A(a, L)$ darstellt. Die explizite Matrixform dieser Operatoren hängt dann von der Wahl der Zustandsbasis ab. Der lineare Raum einer manifest kovarianten Darstellung der iLG ist dadurch definiert, daß es ein vollständiges Basissystem $\{(p \varrho)\}$ gibt, dessen ,Spinorkomponenten“ sich unabhängig vom Impuls $p$ transformieren ${ }^{3}$. Bei Lorentztransformationen $L$ soll also gelten:

$$
\left.A(0, L) \mid p \varrho)=\mid L p \varrho^{\prime}\right) S_{\varrho^{\prime} \varrho}(L) \quad \text { (mit Summenkonvention) }
$$

während sich Translationen einfach als

$$
A(a, 0) \mid p \varrho)=\mid p \varrho) e^{i p a}
$$

${ }^{3}$ Für ihre Fouriertransformierten $(x \varrho)=\int(p \varrho) e^{i p x} d \sigma$ gilt Entsprechendes vom Ort $x . d \sigma=d^{3} \mathbf{p} / p_{0}$ ist das invariante Flächenelement auf dem Massenhyperboloid. Die Zustände werden hier mit runden Klammern geschrieben, damit sie von einer später auftretenden zweiten Zustandssorte unterschieden werden können. 
schreiben. Ihr Charakteristikum ist also eine endlich dimensionale impulsunabhängige Darstellung $S(L)$ der homogenen Lorentzgruppe (Spinordarstellung), die wir hier der Einfachheit halber irreduzibel wählen. Der Grund der Verwendung solcher Darstellungen in der Feldtheorie ist bekannt. Sie sind wegen ihres einfachen Transformationsverhaltens besonders zur Konstruktion relativistisch invarianter lokaler Wechselwirkungen geeignet, obwohl sich andererseits natürlich gezeigt hat, daß damit Konvergenzschwierigkeiten und unphysikalische Züge wie eine indefinite Metrik verbunden sein können. Letzteres ist, wie man vom elektromagnetischen Feld weiß, schon bei freien Feldern möglich.

Wir wollen hier untersuchen, wie man generell solche manifest kovarianten Darstellungen der iLG im wechselwirkungsfreien Falle (KleinGordon-Gleichung) physikalisch interpretieren kann. Dazu müssen wir die Darstellung soweit als möglich in irreduzible unitäre Bestandteile zerlegen, die dann freien Teilchen zugeordnet werden können ${ }^{4}$.

Wir beachten zunächst, daß die Hinzunahme der Impulsabhängigkeit zur Spinordarstellung $S(L)$ gruppentheoretisch gerade dem Übergang zum direkten Produkt

$$
S(L) \otimes T(p)
$$

entspricht, wobei $T(p)$ eine manifest kovariante Darstellung ohne Spinvariable (Skalarfeld) ist (vgl. AhmavaAra [8]). Daß solche direkten Produkte trotz der Irreduzibilität von $S(L)$ im allgemeinen nicht mehr irreduzibel sind, ist bekannt. In unserem Fall wird die Irreduzibilität dadurch zerstört, daß der Impuls eine Richtung im Raumzeitkontinuum auszeichnet. Dies ermöglicht eine eindeutige Zuordnung der homogenen Lorentzgruppe zu einer ihrer Untergruppen - der Wignerschen kleinen Gruppe -, gegenüber der dann die Irreduzibilität nicht mehr gewährleistet ist. Da sie aus den bei festem Impuls noch möglichen Lorentztransformationen besteht, geben ihre Darstellungen zugleich Aufschluß über die Spinfreiheitsgrade der möglichen Teilchen ${ }^{5}$.

Die Reduktion sieht explizit folgendermaßen aus (vgl. WIGner [7]): Man wählt zunächst auf dem Massenhyperboloid eine einfache Normalform $\bar{p}$ des Viererimpulses. Ferner definiert man zu jedem Viererimpuls $p$ eine Lorentztransformation $\Lambda(p)$ die $p$ in $\bar{p}$ überführt. Eine beliebige Lorentztransformation $L$ läßt sich dann nach

zerlegen, wobei

$$
L=\Lambda^{-1}(L p) K \Lambda(p)
$$

$$
K(p, L)=\Lambda(L p) L \Lambda^{-1}(p)
$$

wegen $K \bar{p}=\bar{p}$ zur kleinen Gruppe gehört.

${ }^{4} \operatorname{Im}$ Darstellungsraum $\{(p \varrho)\}$ ist a priori kein Skalarprodukt definiert. Es kann nur dann ein relativistisch invariantes Skalarprodukt eingeführt werden, wenn sich die Darstellung auf eine unitäre oder quasiunitäre Form bringen läßt.

${ }^{5}$ Kleine Gruppen zu verschiedenen Viererimpulsen gleicher Masse sind äquivalent. 
Der wesentliche Schritt besteht nun in einer Anwendung dieser Zerlegung auf den Spinoranteil der manifest kovarianten Darstellung, wobei $p$ mit den Impulseigenwerten der Zustände identifiziert wird ${ }^{6}$. Wir definieren dazu im Zustandsraum eine neue Basis:

$$
\left.|p \varrho\rangle=\mid p \varrho^{\prime}\right) S_{\varrho^{\prime} \varrho}\left(\Lambda^{-1}(p)\right) .
$$

Die $|p \varrho\rangle$ transformieren sich dann wegen

$$
\begin{aligned}
A(L)|p \varrho\rangle & =\mid L p \sigma) S_{\sigma \varrho^{\prime}}(L) S_{\varrho^{\prime} \varrho}\left(\Lambda^{-1}(p)\right) \\
& =\left|L p \sigma^{\prime}\right\rangle S_{\sigma^{\prime} \sigma}(\Lambda(L p)) S_{\sigma \varrho^{\prime}}(L) S_{\varrho^{\prime} \varrho}\left(\Lambda^{-1}(p)\right) \\
& =\left|L p \varrho^{\prime}\right\rangle S_{\varrho^{\prime} \varrho}(K(p, L))
\end{aligned}
$$

nach der Spinordarstellung der kleinen Gruppe, die zwar impulsabhängig ist, dafür aber eine einfachere Struktur hat. Die zu den infinitesimalen Transformationen der kleinen Gruppe gehörigen Drehwinkel $\delta \omega$ bzw. Geschwindigkeiten $\delta \mathbf{u}$ sind nämlich per definitionem durch

d. h. also durch:

$$
\begin{aligned}
\delta \overline{\mathbf{p}} & =\overline{\mathbf{p}} \times \delta \boldsymbol{\omega}+\bar{p}_{0} \delta \mathbf{u}=0 \\
\delta \bar{p}_{0} & =\overline{\mathbf{p}} \cdot \delta \mathbf{u}=0
\end{aligned}
$$

$$
\delta \mathbf{u}=\frac{\delta \boldsymbol{\omega} \times \overline{\mathbf{p}}}{\bar{p}_{0}}
$$

eingeschränkt? ${ }^{7}$ Die infinitesimalen Erzeugenden $\boldsymbol{\Sigma}_{\varrho^{\prime} \varrho}$ bzw. $\boldsymbol{\Pi}_{\varrho^{\prime} \varrho}$ der Darstellung $S(L)$ treten daher bei Transformationen der kleinen Gruppe nur in der Kombination:

$$
\boldsymbol{\Sigma}_{\varrho^{\prime} \varrho} \cdot \delta \boldsymbol{\omega}+\boldsymbol{\Pi}_{\varrho^{\prime} \varrho} \cdot \delta \mathbf{u}=\left(\boldsymbol{\Sigma}_{\varrho^{\prime} \varrho}+\frac{\overline{\mathbf{p}}}{\bar{p}_{0}} \times \boldsymbol{\Pi}_{\varrho^{\prime} \varrho}\right) \cdot \delta \boldsymbol{\omega}
$$

auf; ihre Spinordarstellung läßt sich deshalb bereits aus den drei $^{8}$ infinitesimalen Erzeugenden

$$
\frac{1}{\bar{p}_{0}} \mathbf{W}_{\varrho^{\prime} \varrho}=\boldsymbol{\Sigma}_{\varrho^{\prime} \varrho}+\frac{\overline{\mathbf{p}}}{\bar{p}_{0}} \times \boldsymbol{\Pi}_{\varrho^{\prime} \varrho}
$$

aufbauen. Um das Problem zu konkretisieren, nehmen wir jetzt an, daß die Darstellung $S(L)$ vom Typ $(a, b)$ ist. Das bedeutet bekanntlich, daß sich ihre infinitesimalen Erzeugenden $\boldsymbol{\Sigma}_{\varrho^{\prime} \varrho}$ und $\boldsymbol{\Pi}_{\ell^{\prime} \varrho}$ als

$$
\begin{aligned}
& \boldsymbol{\Sigma}_{Q^{\prime} \varrho} \equiv \boldsymbol{\Sigma}_{\alpha^{\prime} \beta^{\prime} \alpha \beta}=\mathbf{J}_{\alpha^{\prime} \alpha}^{a} \delta_{\beta^{\prime} \beta}+\mathbf{J}_{\beta^{\prime} \beta}^{b} \delta_{\alpha^{\prime} \alpha} \\
& \boldsymbol{\Pi}_{Q^{\prime} \varrho} \equiv \boldsymbol{\Pi}_{\alpha^{\prime} \beta^{\prime} \alpha \beta}=\frac{1}{i}\left(\mathbf{J}_{\alpha^{\prime} \alpha}^{a} \delta_{\beta^{\prime} \beta}-\mathbf{J}_{\beta^{\prime} \beta}^{b} \delta_{\alpha^{\prime} \alpha}\right)
\end{aligned}
$$

${ }^{6}$ Eine ähnliche Zerlegung läßt sich für jede Darstellung der iLG angeben, da die Aufteilung in einen impulsändernden Anteil und einen Spinanteil stets möglich ist [7].

7 Den Fall $\bar{p}_{0}=0$, der für raumartige Viererimpulse auftreten kann, schließen wir hier aus.

${ }^{8}$ Gemeint sind die drei Komponenten des Vektors W. 
schreiben lassen ${ }^{9}$, wobei $\mathbf{J}_{\alpha^{\prime} \alpha}^{a}$ und $\mathbf{J}_{\beta^{\prime} \beta}^{b}$ die infinitesimalen Erzeugenden zweier Darstellungen der Drehgruppe zu den Spins $a$ und $b$ sind. Sie sollen hier hermitesch und in den $z$-Komponenten diagonal gewählt werden, d. h.:

$$
\left(J_{z}^{a}\right)_{\alpha^{\prime} \alpha}=\alpha \delta_{\alpha^{\prime} \alpha}, \quad\left(J_{z}^{b}\right)_{\beta^{\prime} \beta}=\beta \delta_{\beta^{\prime} \beta} .
$$

Einen Gewinn an Übersichtlichkeit bringt dabei die folgende Operatorschreibweise $^{10}$ :

$$
\begin{gathered}
\left.\left.\mid p \alpha^{\prime} \beta^{\prime}\right) S_{\alpha^{\prime} \beta^{\prime} \alpha \beta}\left(\Lambda^{-1}(p)\right) \equiv S\left(\Lambda^{-1}\right) \mid p \alpha \beta\right)=|p \alpha \beta\rangle \\
\left.\left.\left.\left.\mid p \alpha^{\prime} \beta\right) \mathbf{J}_{\alpha^{\prime} \alpha}^{a} \equiv \mathbf{J}^{a} \mid p \alpha \beta\right), \quad \mid p \alpha \beta^{\prime}\right) \mathbf{J}_{\beta^{\prime} \beta}^{b} \equiv \mathbf{J}^{b} \mid p \alpha \beta\right) \\
\left|p \alpha^{\prime} \beta\right\rangle \mathbf{J}_{\alpha^{\prime} \alpha}^{a} \equiv \mathbf{J}^{\prime a}|p \alpha \beta\rangle, \quad\left|p \alpha \beta^{\prime}\right\rangle \mathbf{J}_{\beta^{\prime} \beta}^{b} \equiv \mathbf{J}^{\prime b}|p \alpha \beta\rangle \\
\boldsymbol{\Sigma} \equiv \mathbf{J}^{a}+\mathbf{J}^{b}, \quad \boldsymbol{\Pi} \equiv \frac{1}{i}\left(\mathbf{J}^{a}-\mathbf{J}^{b}\right) \quad \boldsymbol{\Sigma}^{\prime} \equiv \mathbf{J}^{\prime a}+\mathbf{J}^{\prime b}, \quad \boldsymbol{\Pi}^{\prime} \equiv \frac{1}{i}\left(\mathbf{J}^{\prime a}-\mathbf{J}^{\prime b}\right) .
\end{gathered}
$$

Die gestrichenen Operatoren hängen dabei mit den ungestrichenen wegen

$$
\begin{aligned}
\left.J^{\prime} \mid p \varrho\right) & =J^{\prime}\left|p \varrho^{\prime}\right\rangle S_{\varrho^{\prime} \varrho}(\Lambda(p)) \\
& \left.=\mid p \sigma^{\prime}\right) S_{\sigma^{\prime} \sigma}\left(\Lambda^{-1}(p)\right) J_{\sigma^{\prime} \varrho^{\prime}} S_{\varrho^{\prime} \varrho}(\Lambda(p)) \\
& =S(\Lambda) J S(\Lambda) \mid p \varrho)
\end{aligned}
$$

nach der Relation:

$$
J^{\prime}=S^{-1}(\Lambda) J S(\Lambda)
$$

zusammen. Die Basis $|p \alpha \beta\rangle$ diagonalisiert dann die Operatoren $J_{z}^{a}, J_{z}^{b}$,

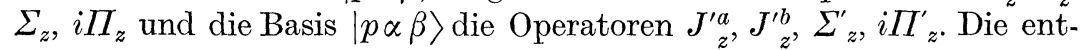
sprechenden Eigenwerte sind $\alpha, \beta, \alpha+\beta$ und $\alpha-\beta$; wir schreiben deshalb auch

$$
\mid p \alpha \beta) \equiv|p h n\rangle, \quad|p \alpha \beta\rangle \equiv|p h n\rangle
$$

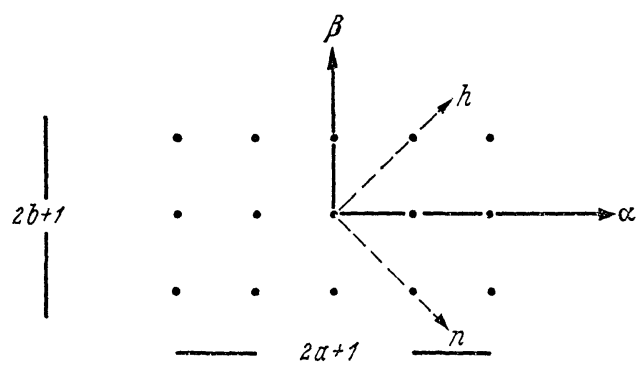

Fig. 1. Eigenwertspektrum von $J_{z}^{\prime a}, J_{z}^{\prime b}, \Sigma_{z}^{\prime}, i \Pi_{z}^{\prime}$

9 Wir ordnen jedem Index $\varrho$ ein Paar $\alpha, \beta$ zu. $\varrho$ durchläuft dann $(2 a+1) \times$ $\times(2 b+1)$ Werte.

${ }_{10}$ Wir müssen beachten, daß die Matrizen $J_{\alpha^{\prime} \alpha}$ usw. je nachdem, ob sie die $|p \alpha \beta\rangle$ oder $\mid p \alpha \beta)$ transformieren, zu verschiedenen linearen Operatoren gehören. 
mit $h=\alpha+\beta, n=\alpha-\beta$. Der Wertebereich von $\alpha, \beta, h$ und $n$ ist in Fig. 1 dargestellt.

Ist nun die Ruhmasse von Null verschieden, so kann $\overline{\mathbf{p}}=0, \bar{p}_{0}=m$ gewählt werden. Die kleine Gruppe geht dann offensichtlich in die Drehgruppe über. Die infinitesimalen Erzeugenden ihrer Spinordarstellung sind nach (5) und (6) durch

$$
\boldsymbol{\Sigma}^{\prime}=\mathbf{J}^{\prime a}+\mathbf{J}^{\prime b}
$$

gegeben; sie ist unitär und zerfällt in irreduzible Bestandteile der Spins

$$
|a+b|, \ldots,|a-b| \text {. }
$$

Im Fall verschwindender Ruhmasse liegen die Verhältnisse jedoch komplizierter. Sie sollen im nächsten Abschnitt untersucht werden.

\section{Darstellungen der kleinen Gruppe und Teilcheninterpretation im Falle verschwindender Ruhmasse}

Bei verschwindender Masse kann der Viererimpuls nur noch auf die Normalform $\overline{\mathbf{p}}=\mathbf{e}_{z}, \bar{p}_{0}=1$ gebracht werden. $\mathbf{e}_{z}$ ist dabei eine feste Raumachse (z-Achse). Dies ändert die Struktur der kleinen Gruppe wesentlich ab und erschwert die Teilcheninterpretation. Nach (5) und (7) folgt nämlich jetzt für ihre infinitesimalen Erzeugenden:

bzw.

$$
\mathbf{W}^{\prime}=\boldsymbol{\Sigma}^{\prime}+\mathbf{e}_{z} \times \boldsymbol{\Pi}^{\prime}
$$

$$
W_{x}^{\prime}=\Sigma_{x}^{\prime}-\Pi_{y}^{\prime}, W_{y}^{\prime}=\Sigma_{y}^{\prime}+\Pi_{x}^{\prime}, W_{z}^{\prime}=\Sigma_{z}^{\prime}
$$

Die Struktur dieser Darstellung wird durchsichtiger, wenn wir das Verhalten von $\Pi_{z}^{\prime}$ unter der kleinen Gruppe betrachten. Anwendung der für die homogene Lorentzgruppe charakteristischen Vertauschungsrelationen von $\boldsymbol{\Sigma}^{\prime}$ und $\boldsymbol{\Pi}^{\prime}$ :

$$
\left[\Sigma_{i}^{\prime}, \Sigma_{k}^{\prime}\right]=i \Sigma_{j}^{\prime},\left[\Pi_{i}^{\prime}, \Pi_{k}^{\prime}\right]=-i \Sigma_{j}^{\prime},\left[\Pi_{i}^{\prime}, \Sigma_{k}^{\prime}\right]=i \Pi_{j}^{\prime} \quad(i, k, j \text { zyklisch })
$$

führt zu:

$$
\begin{aligned}
& {\left[\Pi \Pi_{z}^{\prime}, W_{x}^{\prime}\right]=i \Pi_{y}^{\prime}-i \Sigma_{x}^{\prime}=-i W_{x}^{\prime}} \\
& {\left[\Pi_{z}^{\prime}, W_{y}^{\prime}\right]=-i \Pi_{x}^{\prime}-i \Sigma_{y}^{\prime}=-i W_{y}^{\prime}} \\
& {\left[\Pi_{z}^{\prime}, W_{z}^{\prime}\right]=0 .}
\end{aligned}
$$

Das bedeutet gerade, daß jede beliebige Linearkombination von $W_{x}^{\prime}$ und $W_{y}^{\prime}$ die Eigenwerte von $i \Pi_{z}^{\prime}$ um eins erhöht, während $W_{z}^{\prime}$ sie nicht ändert. Es gilt nämlich:

$$
\begin{aligned}
& i \Pi_{z}^{\prime}\left(\alpha W_{x}^{\prime}+\beta W_{y}^{\prime}\right)|p h n\rangle \\
& =\left(i\left[\Pi_{z}^{\prime}, \alpha W_{x}^{\prime}+\beta W_{y}^{\prime}\right]+n\left(\alpha W_{x}^{\prime}+\beta W_{y}^{\prime}\right)\right)|p h n\rangle \\
& =(n+1)\left(\alpha W_{x}^{\prime}+\beta W_{x}^{\prime}\right)|p h n\rangle .
\end{aligned}
$$


Dadurch vereinfacht sich die Darstellung erheblich. Bei Lorentztransformationen folgt nämlich mit $S(K)=e^{i \omega \cdot w^{\prime}}$ nach (4) und (7):

$$
A(L)|p \varrho\rangle=\left|L p \varrho^{\prime}\right\rangle S_{\varrho^{\prime} \varrho}(K(p, L))=e^{i \omega(p, L) \cdot \mathbf{w}^{\prime}}|L p \varrho\rangle .
$$

Ferner zeigt die Entwicklung dieser Exponentialfunktion, daß $S(K)$ als

$$
e^{i \omega \cdot \mathbf{w}^{\prime}}=e^{i \omega_{z} u_{z}^{\prime}}+F_{\omega}\left(\mathbf{W}^{\prime}\right)
$$

geschrieben werden kann, wobei jedes Potenzreihenglied von $F_{\omega}\left(\mathbf{W}^{\prime}\right)$ mindestens einen Faktor $W_{k}^{\prime}$ oder $W_{y}^{\prime}$ enthält. Damit folgt:

$$
\begin{aligned}
A(L)|p h n\rangle=\left(e^{i \omega_{z} w_{z}^{\prime}}+F_{\omega}(\mathbf{W})\right)|L p h n\rangle & \\
& =|L p h n\rangle e^{i \omega_{z} h}+\sum_{\substack{n^{\prime}>n \\
h^{\prime}}}\left|L p h^{\prime} n^{\prime}\right\rangle \lambda_{h^{\prime} n^{\prime}} .
\end{aligned}
$$

Ein Zustand $|p h n\rangle$ geht also bei Lorentztransformationen in eine Superposition von Zuständen $\left|L p h^{\prime} n^{\prime}\right\rangle$ über, die nur Beiträge zu $n^{\prime} \geqq n$ enthält. Ferner gehen die Anteile zu $n^{\prime}=n$ aus $|L p h n\rangle$ einfach durch Multiplikation mit Phasenfaktoren hervor. Die Verhältnisse sind in Fig. 2 skizziert.
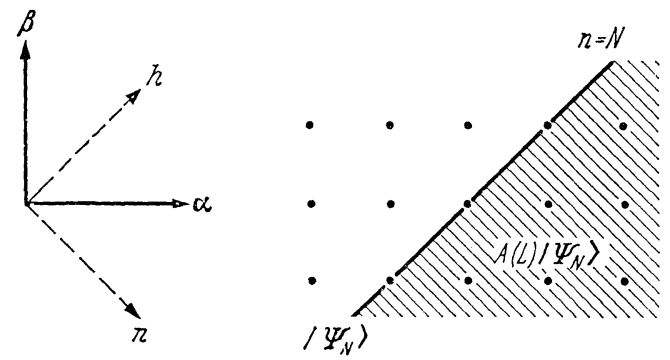

Fig. 2. Die Zustände $\left|\psi_{N}\right\rangle$ werden in den schraffierten Bereich ,hineingestreut”

Damit ist zugleich eine optimale Reduktion der Darstellung erreicht: Der Darstellungsraum zerfällt in eine aufsteigende Folge invarianter Unterräume:

$$
R_{N}=\left\{\sum_{\substack{\gtrless \\ h}}|p h n\rangle \alpha_{h n}, \alpha_{h n} \text { beliebig komplex }\right\} \text { mit } R_{N+1} \subset R_{N} .
$$

Ihre Komplementärräume sind wegen (10) sicher nicht invariant, so daß eine weitere Reduktion unmöglich ist, vgl. HaMmermesh [1].

Ferner kann (11) zu einer - allerdings nicht eindeutigen - Wahrscheinlichkeitsinterpretation benutzt werden. Ein Zustand

$$
\left|\psi_{N}\right\rangle=\sum_{h} \int|p h N\rangle \psi_{h N}(p) d \sigma+\sum_{n>N} \int|p h n\rangle \psi_{h n}(p) d \sigma \in R_{N}
$$


geht nämlich bei Lorentztransformationen in:

$$
A(L)\left|\psi_{N}\right\rangle=\sum_{h}|L p h N\rangle e^{i \omega_{z}(p, L)} \psi_{h N}(p) d \sigma+\underset{\substack{h>N \\ h}}{\sum_{n}}|L p h n\rangle \psi_{h n}^{\prime}(p) d \sigma
$$

über, da sich die zum niedrigsten Index $n=N$ gehörigen Anteile bis auf Phasenfaktoren reproduzieren; die Koeffizienten $\psi_{h N}(p)$ können somit als Wahrscheinlichkeitsamplituden aufgefaßt werden. Die entsprechenden Skalarprodukte lassen sich in einfacher Weise durch Projektionsoperatoren $T^{N}$ formulieren, die auf die zum Index $N$ gehörigen Zustände projizieren :

$$
\Gamma^{N}|p h n\rangle=|p h n\rangle \delta_{n N} .
$$

Die Bedingung $|\psi\rangle \in R_{N}$ bzw. $\psi_{h n}=0$ für $n<N$ läßt sich dann durch:

$$
0=\Gamma^{-(a+b)}|\psi\rangle=\Gamma^{-(a+b)+1}|\psi\rangle=\cdots=\Gamma^{N-1}|\psi\rangle
$$

ausdrücken ${ }^{11}$. Wir definieren ferner eine Bilinearform $\langle\chi \mid \psi\rangle$ durch

was nach (12):

$$
\left\langle p h n \mid p^{\prime} h^{\prime} n^{\prime}\right\rangle=p_{0} \delta\left(\mathbf{p}-\mathbf{p}^{\prime}\right) \delta_{h h^{\prime}} \delta_{n n^{\prime}}
$$

$$
\langle\chi \mid \psi\rangle=\sum_{h n} \int \chi_{h n}^{*}(p) \psi_{h n}(p) d \sigma
$$

impliziert. Sie ist offensichtlich nicht lorentzinvariant, da die Spinordarstellung $\left\{S_{\varrho^{\prime} \varrho}(K(p, L))\right\}$ nicht unitär ist. Nach (13) ist jedoch, falls die Bedingungen (14) gelten, die Bilinearform

$$
\sum_{h} \int \chi_{h N}^{*}(p) \psi_{h N}(p) d \sigma=\left\langle\chi\left|\Gamma^{N}\right| \psi\right\rangle
$$

relativistisch invariant, da sie nur die zum Index $N$ gehörigen Amplituden enthält.

(14) und (16) führen somit zu einer ganzen Klasse möglicher Wahrscheinlichkeitsinterpretationen, die sich durch verschiedene Werte des Index $N$ unterscheiden. Auf diese Weise wird es möglich, mit einem manifest kovarianten Feld des Typs $(a, b)$ Teilchen der Helizitäten $|a+b|, \ldots,-|a+b| \mathrm{zu}$ beschreiben. Diese Interpretation hat die Eigenart, daß es gewisse Zustände $|\psi\rangle \in R_{N+1}$ gibt, die zum Skalarprodukt nichts beitragen, und folglich nur indirekt beobachtbar sind. Sie können deshalb als Eichzusätze aufgefaßt werden. Wie Abb. 2 zeigt, verringert sich ihre Zahl mit wachsender Zahl der Nebenbedingungen (14) und verschwindet schließlich, wenn $N$ seinen Maximalwert $N=a+b$ erreicht. Der Zustandsraum wird dann irreduzibel und beschreibt ein Teilchen der Helizität $a-b$. Dieser Fall wird von S. WeInberg [4] erörtert.

Bevor wir zur Diskussion konkreter Beispiele kommen, formen wir die Bedingungen (14) und (16) etwas um. Sie bekommen eine hand-

\footnotetext{
11 Sie bilden demnach zusammen ein relativistisch invariantes System.
} 
lichere Form, wenn wir sie in den ungestrichenen Operatoren formulieren, die nach (7) unmittelbar auf die in der Feldtheorie geläufigeren manifest kovarianten Zustände wirken. Wir beachten zunächst, daß sich die Projektionsoperatoren $\Gamma^{N}$ als

$$
\Gamma^{N}=\sum_{K} \gamma_{K}^{N} \Pi_{z}^{\prime K}
$$

schreiben lassen, da sie auf die zu $N$ gehörigen Eigenwerträume von $\Pi_{z}^{\prime}$ projizieren. Für den Operator $\Pi_{z}^{\prime}$ gilt ferner nach (8):

$$
\Pi_{z}^{\prime}=S \stackrel{-1}{-1} \Pi_{z} S(\Lambda) .
$$

Darin ist $\Lambda(p)$ eine Lorentztransformation, die $p$ auf $\bar{p}$ abbildet. Wir setzen sie aus einer Raumdrehung, die den Impuls in die $z$-Richtung dreht, und einer Lorentztransformation, die seinen Betrag auf 1 transformiert, zusammen. Für ihre Spinordarstellung $S(\Lambda)$ gilt dann:

$$
S(\Lambda)=e^{-i \theta \Pi_{z}} e^{-i \omega \cdot \Sigma},
$$

wobei der Drehwinkel $\boldsymbol{\omega}$ durch

$$
\boldsymbol{\omega}=\frac{\mathbf{e}_{z} \times \mathbf{p}}{|\mathbf{p}| \sin \vartheta}, \quad \cos \vartheta=\frac{p_{z}}{\mid \mathbf{p} !}
$$

und $\theta$ durch:

$$
\mathbf{1}=p_{0} \cosh \theta+|\mathbf{p}| \sinh \theta, \quad \text { d. h. } \quad \theta=-\ln p_{0}
$$

bestimmt ist. Damit folgt sofort:

sowie

$$
\Pi_{z}^{\prime}=e^{i \omega \cdot \Sigma} \Pi_{z} e^{-i \omega \cdot \Sigma}=\frac{\boldsymbol{\Pi} \cdot \mathbf{p}}{|\mathbf{p}|}
$$

$$
\Gamma^{N}=\sum_{K} \gamma_{K}^{N}\left(\frac{\boldsymbol{\Pi} \cdot \mathbf{p}}{|\mathbf{p}|}\right)^{K} .
$$

Wir berechnen ferner das Skalarprodukt zweier manifest kovarianter Zustände. Nach (3), (15) und (16) folgt:

und nach (17) und (18) ${ }^{12}$ :

$$
\begin{aligned}
\left(p_{1} \varrho_{1}\left|\Gamma^{N}\right| p_{2} \varrho_{2}\right) & =\left(p_{1} \varrho_{1} \mid p_{2} \varrho\right) \Gamma_{\varrho^{\prime} \varrho_{2}}^{N} \\
& =\left\langle p_{1} \varrho_{1}^{\prime} \mid p_{2} \varrho^{\prime}\right\rangle S_{\varrho_{1} \varrho_{1}^{\prime}}^{+} S_{\varrho^{\prime} \varrho} I_{\varrho \varrho_{2}}^{N} \\
& =p_{0} \delta\left(\mathbf{p}-\mathbf{p}^{\prime}\right)\left(S^{+} S \Gamma^{N}\right)_{\varrho_{1} \varrho_{2}}
\end{aligned}
$$

$$
S S^{+} \Gamma^{N}=e^{i \boldsymbol{\omega} \cdot \Sigma} e^{-2 i \theta \Pi_{z}} e^{-i \boldsymbol{\omega} \cdot \Sigma} \Gamma^{N}=e^{-2 i \theta} \frac{\boldsymbol{\Pi} \cdot \mathbf{p}}{|\mathbf{p}|} \Gamma^{N} .
$$

Da $\Gamma^{N}$ auf den Unterraum projiziert, der zum Eigenwert $N$ von $i \cdot \frac{\boldsymbol{I} \cdot \mathbf{p}}{|\mathbf{p}|}$ gehört, ergibt sich schließlich:

$$
S^{+} S \Gamma^{N}=e^{-2 \theta N} \Gamma^{N}=p_{0}^{2 N} \Gamma^{N}
$$

${ }^{12}$ Wir benutzen dabei die Hermitezität von $\boldsymbol{\Sigma}_{\alpha \beta}$ und $i \boldsymbol{\Pi}_{\alpha \beta}$. 
bzw.

$$
\left(p_{1} \varrho_{1}\left|\Gamma^{N}\right| p_{2} \varrho_{2}\right)=p_{0} \delta\left(\mathbf{p}_{1}-\mathbf{p}_{2}\right) p_{0}^{2 N} \sum_{K} \gamma_{K}^{N}\left(\left(\frac{\boldsymbol{I} \cdot \mathbf{p}}{|\mathbf{p}|}\right)^{K}\right)_{\varrho_{1} \varrho_{2}} .
$$

Wir wollen diese Methoden jetzt am Beispiel des $\left(\frac{1}{2}, \frac{1}{2}\right)$ Feldes (elektromagnetische Potentiale) und $\left(\frac{1}{2}, 0\right)$ Feldes (Neutrino) illustrieren.

1. Das $\alpha, \beta$ Diagramm des Vektorpotentials $\left(a=\frac{1}{2}=b\right)$ ist in Fig. 3 dargestellt.

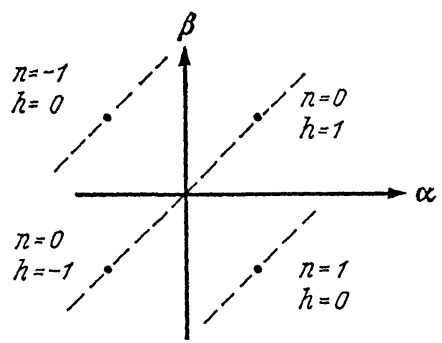

Fig. 3. Eigenwertspektrum von $J_{z}^{\prime a}, J_{z}^{\prime b}, \Sigma_{z}^{\prime}, i \Pi_{z}^{\prime}$ beim Vektorpotential

Der Operator $i \Pi_{z}^{\prime}=i \frac{\boldsymbol{\Pi} \cdot \mathbf{p}}{|\mathbf{p}|}$ hat hier die Eigenwerte $n=-1,0,1$ und wir bekommen die Projektionsoperatoren:

$$
\Gamma^{ \pm 1}=\frac{1}{2}\left(-\Pi_{z}^{\prime 2} \pm i \Pi_{z}^{\prime}\right), \quad \Gamma^{0}=1+\Pi_{z}^{\prime 2} .
$$

Die Zustände:

$$
\begin{aligned}
& \left.\left|p t_{1}\right\rangle=\frac{1}{\sqrt{2}}(|p l l l l| l p-10\rangle\right) \\
& \left|p t_{2}\right\rangle=\frac{1}{i \sqrt{2}}(|p \quad 10\rangle-|p-10\rangle) \\
& |p l\rangle=\frac{1}{\sqrt{2}}(|p 0-1\rangle+|p 01\rangle) \\
& |p s\rangle=\frac{1}{\sqrt{2}}(|p 0-1\rangle-|p 01\rangle)
\end{aligned}
$$

sind dem Vierervektorcharakter der Darstellung besser angepaßt. Sie beschreiben transversale, longitudinale und skalare Photonen und transformieren sich unter den Projektionsoperatoren $\Gamma^{ \pm 1}, \Gamma^{0}$ nach:

$$
\begin{aligned}
\Gamma^{ \pm 1}\left|p t_{1,2}\right\rangle=0, \quad \Gamma^{ \pm 1}|p l\rangle & =\frac{1}{2}(|p l\rangle \mp|p s\rangle) \\
\Gamma^{ \pm 1}|p s\rangle & =\frac{1}{2}(|p s\rangle \mp|p l\rangle) \\
\Gamma^{0}\left|p t_{1,2}\right\rangle=\left|p t_{1,2}\right\rangle, \quad \Gamma^{0}|p l\rangle & =0 \\
\Gamma^{0}|p s\rangle & =0 .
\end{aligned}
$$


Nach der vorangegangenen Analyse haben wir dann drei Fälle zu unterscheiden:

a) Keine Nebenbedingung; Skalarprodukt $\left\langle\chi\left|\Gamma^{-1}\right| \psi\right\rangle$. Da $\Gamma^{-1}|\psi\rangle=0$ mit der Lorentzbedingung identisch ist, tragen hier nur Zustände zum Skalarprodukt bei, die der Lorentzbedingung nicht genügen, während transversale Photonen und Mischungen longitudinaler und skalarer Photonen, die ihr genügen, als Eichteilchen auftreten.

b) Nebenbedingung $\Gamma^{-1}|\psi\rangle=0$; Skalarprodukt $\left\langle\chi\left|\Gamma^{0}\right| \psi\right\rangle$. Wir haben es dann mit der üblichen Interpretation der elektromagnetischen Potentiale zu tun, wobei das Skalarprodukt so geschrieben ist, daß die longitudinalen und skalaren Photonen explizit herausprojiziert werden. (Beim Skalarprodukt von Gupta und Bleuler, das dazu äquivalent ist, heben sich diese Anteile auf, sofern die Lorentzbedingung erfüllt ist.)

c) Nebenbedingungen $\Gamma^{-1}|\psi\rangle=0=\Gamma^{0}|\psi\rangle$; Skalarprodukt $\left\langle\chi\left|\Gamma^{+1}\right| \psi\right\rangle$ $=\langle\chi \mid \psi\rangle$. Es handelt sich dabei um elektromagnetische Potentiale ohne transversale Photonen (Eichfelder).

Wir bekommen also hier die bekannten Vektorpotentialtypen zurück. Jeder dieser Feldtypen läßt eine relativistisch invariante Wahrscheinlichkeitsinterpretation zu.

2. Im Falle des $\left(\frac{1}{2}, 0\right)$ Feldes bekommen wir das in Fig. 4 skizzierte $\alpha, \beta$ Diagramm.

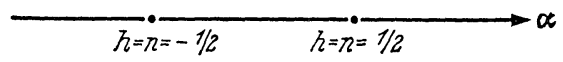

Fig. 4. Spektrum von $J_{z}^{\prime a}, J_{z}^{\prime b}, \Sigma_{z}^{\prime}, i \Pi_{z}^{\prime}$ beim $\left(\frac{1}{2}, 0\right)$ Feld

Die $J_{\alpha^{\prime} \alpha}^{a}$ sind hier bis auf einen Faktor $\frac{1}{2}$ mit den Paulimatrizen $\boldsymbol{\sigma}_{\alpha^{\prime} \alpha}$ identisch, während die $\mathbf{J}_{\beta^{\prime} \beta}^{b}$ verschwinden. Nach (7) gilt deshalb $i \boldsymbol{I}=\frac{\mathbf{1}}{\mathbf{2}} \boldsymbol{\sigma}$ und wir bekommen die Projektionsoperatoren:

$$
\Gamma^{ \pm 1 / 2}=\left(\frac{1}{2} \pm i \frac{\boldsymbol{\Pi} \cdot \mathbf{p}}{|\mathbf{p}|}\right)=\frac{1}{2}\left(1 \pm \frac{\boldsymbol{\sigma} \cdot \mathbf{p}}{|\mathbf{p}|}\right) .
$$

Dies führt zu der Aufteilung:

a) Keine Nebenbedingung; Skalarprodukt

$$
\left\langle\chi\left|\Gamma^{-1 / 2}\right| \psi\right\rangle=\frac{1}{2}\left\langle\chi\left|1-\frac{\boldsymbol{\sigma} \cdot \mathbf{p}}{|\mathbf{p}|}\right| \psi\right\rangle .
$$

Zum Skalarprodukt tragen dann nur Neutrinozustände der Helizität $-\frac{1}{2}$ bei, während die Weylschen Neutrinos $\left(h=\frac{1}{2}\right)$ als Eichteilchen auftreten. Es ist demnach möglich, durch ein $\left(\frac{1}{2}, 0\right)$ Feld auch ein 
Neutrino der Helizität - $\frac{1}{2}$ zu beschreiben, wenn man - wie bei den elektromagnetischen Potentialen - das Auftreten von Eichteilchen in Kauf nimmt.

b) Nebenbedingung $\Gamma^{-1 / 2}|\psi\rangle=0=\left(1-\frac{\boldsymbol{\sigma} \cdot \mathbf{p}}{|\mathbf{p}|}\right)|\psi\rangle$ und Skalarprodukt $\left\langle\psi\left|\Gamma^{-1 / 2}\right| \psi\right\rangle=\langle\chi \mid \psi\rangle$. Dies ist die übliche Interpretation eines $\left(\frac{1}{2}, 0\right)$ Feldes, das der Weyl-Gleichung genügt. Es beschreibt ein Neutrino der Helizität $+\frac{1}{2}$.

Die beiden Beispiele verdeutlichen zwei für die manifest kovarianten Darstellungen der iLG zur Masse Null typische Eigenschaften: Die Freiheit in der physikalischen Interpretation und das Auftreten von Eichteilchen. Ferner zeigen sie, wie man jeweils die Nebenbedingungen zu wählen hat, wenn man Teilchen der Helizitäten $|a+b|, \ldots,-|a+b|$ beschreiben will.

Umgekehrt führt somit die Aufgabe der Irreduzibilität des Zustandsraums zu einer Erweiterung der Klasse der Felder, die ein Teilchen der Helizität $h$ beschreiben. Sie sind jetzt nicht mehr durch $a-b=h$, sondern nur noch durch $|a+b| \geqq|h|\left(\frac{a+b}{h}\right.$ ganz $)$ eingeschränkt.

Vom gruppentheoretischen Standpunkt sind diese Felder alle mehr oder weniger gleichberechtigt. Es hat jedoch den Anschein, daß es für jedes Teilchen ganz bestimmte Felder und Nebenbedingungen gibt, mit denen sich seine Wechselwirkungen am einfachsten formulieren lassen. Jedenfalls hat sich bisher für das Photon das Vektorpotential mit Lorentzbedingung und für das Neutrino das $\left(\frac{1}{2}, 0\right)$ Feld mit Weylgleichung am besten bewährt.

\section{Der Grenziall $m \rightarrow 0$}

Wir wollen abschließend das Zustandekommen dieser Eigentümlichkeiten der manifest kovarianten Darstellungen an einem Grenzübergang $m \rightarrow 0$ diskutieren. Im Falle $m \neq 0$ kann man $\bar{p}$ auf die Form $\overline{\mathbf{p}}=0$, $\bar{p}_{0}=m$ bringen, und $\Lambda(p)$ wird zur Transformation auf Ruhe. Thre Spinordarstellung läßt sich bekanntlich als

$$
S(\Lambda)=e^{-i \Theta \frac{\Gamma \cdot \mathbf{p}}{|\mathbf{p}|}}, \theta=-\operatorname{arctanh} \frac{|\mathbf{p}|}{p_{0}}=-\ln \frac{p_{0}+|\mathbf{p}|}{m}
$$

schreiben [9]. Die Zustände

$$
\left.|p h n\rangle=e^{-i \Theta \frac{\Pi \cdot \mathbf{p}}{|\mathbf{p}|}} \mid p h n\right)
$$

bilden dann nach (5) und (6) eine unitäre Basis und ermöglichen eine einfache Teilcheninterpretation. (19) vereinfacht sich offensichtlich für 
Eigenzustände von $\frac{\boldsymbol{\Pi} \cdot \mathbf{p}}{|\mathbf{p}|}$. Liegt insbesondere der Impuls in $z$-Richtung, so gilt $\frac{\boldsymbol{\Pi} \cdot \mathbf{p}}{|\mathbf{p}|}=\Pi_{z}$ und es folgt nach (7):

$$
\mid p h n)=\left(\frac{p_{0}+|\mathbf{p}|}{m}\right)^{n}|p h n\rangle .
$$

Dieser Zusammenhang führt jedoch im Grenzfalle $m=0 \mathrm{zu}$ Unendlichkeiten, deren Ordnungen vom Eigenwert $n$ abhängen. Wir wollen jetzt zeigen, daß gerade diese Abhängigkeit für die Eigentümlichkeiten der manifest kovarianten Darstellungen zur Masse Null verantwortlich ist. Dazu beschränken wir uns zunächst auf Zustände $|p h N\rangle$ mit einem festen Eigenwert $n=N$ und multiplizieren (20) mit dem lorentzinvarianten Faktor $m^{N 13}$ :

$$
\mid p h n)=\left(p_{0}+|\mathbf{p}|\right)^{n}|p h n\rangle m^{N-n} .
$$

Die $\mid p h N)$ bleiben dann für $m \rightarrow 0$ endlich. Das Verfahren ist jedoch nur dann sinnvoll, wenn dadurch auch in anderen Bezugssystemen Unendlichkeiten vermieden werden. Zur Klärung dieser Frage betrachten wir das Verhalten der $|p h N\rangle$ unter Lorentztransformationen $L$. Ihr Impulseigenwert $p$ geht dabei in $p^{\prime}=L p$ über, während sich die zugehörige Drehung im Spinraum nach (2) berechnet. Für infinitesimale Transformationen kann das Ergebnis leicht angegeben werden. Man weiß nämlich aus der speziellen Relativitätstheorie, daß einer infinitesimalen Lorentztransformation im Laborsystem mit der Geschwindigkeit $\delta \mathbf{u}$ im Ruhsystem eine Drehung um den Winkel $\delta \boldsymbol{\omega}_{s}=\frac{\mathbf{p} \times \delta \mathbf{u}}{p_{0}+m}$ entspricht ${ }^{14}$. Es gilt demnach

$$
|p h N\rangle^{\prime}=A(\delta \mathbf{u})|p h N\rangle=\left(\mathbf{1}-i \delta \boldsymbol{\omega}_{s} \cdot \boldsymbol{\Sigma}\right)\left|p^{\prime} h N\right\rangle .
$$

Die $|p h N\rangle^{\prime}$ werden im allgemeinen keine Eigenzustände von $\frac{\boldsymbol{\Sigma} \cdot \mathbf{p}^{\prime}}{\left|\mathbf{p}^{\prime}\right|}$ und $i \frac{\boldsymbol{\Pi} \cdot \mathbf{p}^{\prime}}{\left|\mathbf{p}^{\prime}\right|}$ sein; letztere gehen vielmehr aus den $\left|p^{\prime} h N\right\rangle$ durch eine Drehung:

$$
\underline{\left|p^{\prime} h N\right\rangle}=\left(1-i \delta \boldsymbol{\omega}_{p} \cdot \boldsymbol{\Sigma}\right)\left|p^{\prime} h N\right\rangle
$$

im Spinraum hervor, wobei der Winkel $\delta \boldsymbol{\omega}_{p}=\frac{p_{0}}{\mathbf{p}^{2}} \mathbf{p} \times \delta \mathbf{u}$ die Änderung der Impulsrichtung beschreibt. Ihr Zusammenhang mit den $|p h N\rangle^{\prime}$ wird klar, wenn wir die Transformation $\left(1-i \delta \boldsymbol{\omega}_{s} \cdot \boldsymbol{\Sigma}\right)$ nach:

$$
1-i \delta \boldsymbol{\omega}_{s} \cdot \boldsymbol{\Sigma}=\left(1-i\left(\delta \boldsymbol{\omega}_{s}-\delta \boldsymbol{\omega}_{p}\right) \cdot \boldsymbol{\Sigma}\right)\left(1-i \delta \boldsymbol{\omega}_{p} \cdot \boldsymbol{\Sigma}\right)
$$

${ }^{13}$ Die so umnormierten manifest kovarianten Zustände werden wieder mit $|p h N|$ bezeichnet.

${ }^{14}$ Dies ist bekanntlich auch der Grund für die sog. Thomaspräzession [10] 
zerlegen. (22) geht dann in:

$$
\begin{aligned}
|p h N\rangle^{\prime}= & \left(1-i\left(\delta \boldsymbol{\omega}_{s}-\delta \boldsymbol{\omega}_{p}\right) \cdot \boldsymbol{\Sigma}\right) \underline{\left|p^{\prime} h N\right\rangle} \\
= & \left(1+i \frac{m}{\mathbf{p}^{2}}(\mathbf{p} \times \delta \mathbf{u}) \cdot \boldsymbol{\Sigma}\right) \underline{\left|p^{\prime} h N\right\rangle} \\
& \left(1+i \frac{m}{\mathbf{p}^{2}}\left(\delta u_{x} \Sigma_{y}-\delta u_{y} \Sigma_{x}\right)\right) \underline{\left|p^{\prime} h N\right\rangle}
\end{aligned}
$$

über. Da sich andererseits die Operatoren $\Sigma_{x}$ und $\Sigma_{y}$ nach (7) linear aus den Schiebeoperatoren $J_{ \pm}^{a}=J_{x}^{a} \pm i J_{y}^{a}$ und $J_{ \pm}^{b}=J_{x}^{b} \pm i J_{y}^{b}$ kombinieren, enthalten die $|p h N\rangle^{\prime}$ außer $\underline{\left|p^{\prime} h N\right\rangle}$ nur Beimischungen:

$$
|\varphi \pm\rangle=\underline{\left|p^{\prime} h^{\prime} N \pm 1\right\rangle} \frac{m}{\mathbf{p}^{2}} \delta u
$$

der Größenordnung $m$. Beim Übergang zur manifest kovarianten Basis haben wir sie nach (21) mit den Faktoren:

$$
m^{\mp 1}\left(p_{0}+|\mathbf{p}|\right)^{N \pm 1}
$$

zu multiplizieren und bekommen zu den $(p h N)^{\prime}$ die Beiträge ${ }^{15}$

$$
\begin{aligned}
\mid \varphi \pm)=|\varphi \pm\rangle\left(p_{0}+|\mathbf{p}|\right)^{N \pm 1} m^{\mp 1} & \\
& =\underline{\left|p^{\prime} h^{\prime} N \pm 1\right\rangle} \frac{\left(p_{0}+|\mathbf{p}|\right)^{N_{ \pm}}}{\mathbf{p}^{2}} \delta u m m^{\mp 1} .
\end{aligned}
$$

Im Grenzfalle $m \rightarrow 0$ verschwinden somit sowohl die Beiträge $|\varphi \pm\rangle$ zur unitären Basis, als auch die Beiträge $(\varphi-)$ zur manifest kovarianten Basis, während sich die Anteile $|\varphi+\rangle$ durch den Faktor $\frac{1}{m}$ in (24) so verstärken, daß die $(\varphi+)$ auch bei $m=0$ endlich bleiben. Die unitären Darstellungen zerfallen somit in irreduzible Bestandteile, von denen jeder durch ein Indexpaar $(h, n)$ gekennzeichnet werden kann, nicht aber die manifest kovarianten Darstellungen. Bei infinitesimalen Lorentztransformationen treten vielmehr zu den Zuständen $\mid p h N)$ endliche Beiträge mit dem Index $N+1$ hinzu.

\section{Literatur}

[1] Hammermesh, M.: Group theory. Reading, Mass.: Addison Wesley 1962.

[2] Joos, H.: Fortschr. Phys. 10, 65 (1962).

[3] Pursey, D. L.: Ann. Phys. 32, 157 (1965).

[4] Weinberg, S.: Phys. Rev. 134, B 882 (1964).

[5] Fronsdal, C.: Phys. Rev. 113, 1367 (1959).

[6] Shirkov, M.: Sov. Phys. JETP 6, 919, 929 (1958).

[7] Wigner, E. P.: Ann. Math. 40, 149 (1939).

[8] Ahmavaara, Y.: Ann. Acad. Sci. Fenn. Ser. A VI, No. 106 (1962).

[9] Messiah, A.: Quantum mechanics II, Amsterdam: North Holl. Publ. Comp. 1962.

[10] Thomas, L. H.: Phil. Mag. 3, 1 (1927).

15 Die Unterscheidung zwischen $p$ und $p^{\prime}$ ist hier belanglos, da sie sich erst in der Ordnung $(\delta u)^{2}$ bemerkbar macht. 